\title{
EL EMPRESARIO TURÍSTICO ESPAÑOL: FORMACIÓN, MOTIVACIONES Y ORIENTACIÓN EMPRENDEDORA ${ }^{1}$
}

\author{
$M^{\text {a }}$ José Rodríguez-Gutiérrez ${ }^{2}$ \\ mjrodri@us.es \\ Isidoro Romero ${ }^{3}$ \\ isidoro@us.es \\ Pilar Tejada ${ }^{4}$ \\ ptejada@us.es \\ Universidad de Sevilla \\ Departamento de Economía Aplicada I
}

(Fecha envío: 11/11/16 - Fecha aceptación: 10/12/16)

\section{Resumen}

Las características personales de los empresarios condicionan el funcionamiento y los resultados de las pequeñas y medianas empresas a nivel microeconómico, determinando junto con otros factores (organizacionales, económicos y del entorno) su calidad emprendedora. A su vez, la calidad emprendedora en un ámbito territorial específico determina la capacidad del tejido empresarial para impulsar el crecimiento y el desarrollo económicos en dicho territorio. Por lo tanto, resulta de gran interés profundizar en el estudio de las características personales de los empresarios de las pymes en un sector como el turístico, que es clave para la economía española y donde la presencia de pymes es abrumadora.

Este trabajo presenta resultados empíricos obtenidos a partir de una encuesta dirigida a 1.119 pymes turísticas españolas, de la que se derivan muestras representativas de las tres principales actividades turísticas: hotelería, intermediación turística y restauración. El trabajo proporciona un perfil personal de los pequeños y medianos empresarios turísticos españoles en relación con aspectos como su nivel formativo y experiencia previa, sus motivaciones, actitudes y orientación emprendedora, entre otros aspectos.

\section{Palabras clave}

empresario, turismo, PYME

\section{Abstract}

The personal characteristics of entrepreneurs condition the activity and results of small and mediumsized enterprises at the micro level determining, together with other factors (organizational, economic and environmental), their entrepreneurial quality. In turn, the entrepreneurial quality in a specific geographical area determines the ability of the businesses to drive growth and economic development in the territory.

Therefore, it is of great interest to deepen the study of personal characteristics of the entrepreneurs running SMEs in the tourism sector, a key sector in the Spanish economy in which the presence of SMEs is overwhelming.

This paper presents some empirical results obtained from a survey of 1,119 Spanish tourism SMEs, which representative samples of the three main tourist activities -hotels, tourist mediation and restoration- were obtained. This work provides a personal profile of entrepreneurs running tourism SMEs in relation to aspects such as their education level and previous experience, motivations, entrepreneurial attitudes and orientation, among others.

\footnotetext{
${ }^{1}$ Este artículo es parte del Proyecto "Pymes turísticas, Cadenas de Valor Globales e Innovación" (ECO2013-42889-P), financiado por el Ministerio de Economía y Competitividad (Plan Nacional de Investigación Científica y Técnica e Innovación).

${ }^{2}$ Dra en Economía por la Universidad de Sevilla

${ }^{3}$ Dr. en Economía por la Universidad de Sevilla

${ }^{4} \mathrm{Dr}^{\mathrm{a}}$ en Economía por la Universidad de Sevilla
} 


\section{Key-words}

entrepreneur, tourism, SME

\section{INTRODUCCIÓN}

España se ha consolidado en las últimas décadas como un destino principal en el mercado turístico mundial. Según los datos más recientes, proporcionados por la Organización Mundial de Turismo (OMT, 2016), en 2015 figura como el tercer país del mundo que más ingresos obtiene del turismo internacional y el primero de Europa (57.000 millones de dólares), así como el tercero por llegadas de turistas internacionales (68,1 millones). Además, estas posiciones no se registran puntualmente, sino que su presencia es constante entre los cinco primeros puestos en ambos rankings mundiales.

Por todo ello, es indudable, que la actividad turística ha sido uno de los motores del proceso de desarrollo económico en España a lo largo de los últimos cincuenta años (Cuadrado y López, 2014, Brida, Pereyra, Such, Devesa y Zapata Aguirre, 2008). No obstante, la atomización del tejido empresarial turístico es especialmente acusada. Según los datos del Directorio Central de Empresas (DIRCE), más del 99\% de las empresas turísticas son pymes. En concreto, la presencia de microempresas en este sector supera el 95\% (INE, 2016).

El estudio del factor empresarial en el ámbito de las actividades turísticas empezó a ganar cierto impulso en la década de los noventa debido al creciente interés de académicos y políticos por conocer las características, estrategias adoptadas y resultados obtenidos por las pymes en el turismo (Page, Forer y Lawton, 1999). Este interés empieza a desarrollarse a partir de la celebración de diferentes conferencias internacionales (Morrison, 2003), y va a fomentar la creación de redes de investigación y debate que favorecen el intercambio de información y conocimientos sobre el papel de las pymes en el turismo (Thomas, Shaw y Page, 2011). Empieza así a formarse un cuerpo de conocimientos teóricos sustentado, por un lado en manuales para estudiantes de cursos sobre emprendedores y pequeñas empresas turísticas (Morrison, Rimmington y Williams, 1999; Thomas, 1998) y, por otro, en un conjunto de publicaciones en las que se reconoce la importancia de estas empresas para la creación de empleo (Wanhill, 2000), la competitividad de los destinos turísticos (Novelli, Schmitz, y Spencer, 2005; OECD, 2008), el desarrollo de un turismo sostenible (Fuller, Buultjens, y Cummings, 2005), y en definitiva, para el crecimiento y desarrollo económico (Andriotis, 2002; Wanhill, 1996). A pesar de este prometedor inicio, el progreso en este campo de investigación ha sido excesivamente lento e irregular, por lo que aún se conoce poco acerca de los procesos empresariales en el turismo y su impacto económico, el papel que juega el empresario turístico y las similitudes o diferencias en los resultados de las pymes y las características de los empresarios en los diferentes subsectores turísticos (Thomas et al., 2011; Smith, 2006; Reichel y Haber, 2005).

El objetivo de esta comunicación es profundizar en el estudio de la capacidad emprendedora de los empresarios de las pymes del sector turístico, un sector de actividad clave para la economía española. Se trata de un estudio de carácter empírico, con base en una amplia muestra de pymes españolas representativas de tres de las principales actividades turísticas: hotelería, intermediación turística y restauración. El trabajo describe aquellas características personales de los pequeños y medianos empresarios turísticos españoles que condicionan su dinamismo emprendedor y, en consecuencia, el funcionamiento y los resultados de sus empresas.

El trabajo se estructura en cinco apartados. Tras esta introducción, en la segunda sección se examinan las principales aportaciones en relación con el marco teórico. A continuación, en el tercer apartado se describe la metodología que se ha utilizado para la elaboración de la parte empírica. El cuarto apartado muestra los resultados obtenidos en este estudio: un perfil del empresario turístico español. Por último, se presentan algunas conclusiones finales.

\section{ESTADO DE LA CUESTIÓN Y MARCO TEÓRICO}

El escaso desarrollo de las investigaciones sobre las pymes y el empresario en el ámbito del turismo contrasta con el alcanzado en otros sectores de actividad, y se explica, entre otras razones, porque la mayor parte de los estudios realizados hasta la fecha se han desarrollado de forma aislada, al margen de los estudios previos, y en referencia a ámbitos geográficos y actividades turísticas muy concretas. Ello entorpece la generalización de sus conclusiones y ha dificultado la consolidación de un marco teórico. La compleja naturaleza de las actividades turísticas, junto con la necesidad de aplicar una perspectiva de análisis interdisciplinar, complican aún más la formación de este marco teórico. No obstante, lo anterior, se han producido algunos avances a partir de diversos trabajos, fundamentalmente empíricos y de carácter descriptivo, que en muchos casos extrapolan al sector turístico los resultados obtenidos para las empresas de otros sectores de actividad (Thomas, 2000; Page et al., 1999).

Aunque el número de publicaciones en este campo es aún escaso, los temas objetos de análisis han sido muy variados, destacando, entre otros, la conceptualización de pymes en el sector turístico y la necesidad de identificar de forma adecuada los pequeños negocios en el turismo; las características de las empresas 
turísticas; las motivaciones de sus emprendedores; y los procesos de gestión empresarial e innovación en las empresas turísticas (Ateljevic y Li, 2009). La mayoría de estos estudios se centran en el sector de la hotelería y tienen un marcado carácter cualitativo, siendo muy reducido el número de trabajos basados en datos primarios.

A este respecto, se ha sugerido la necesidad de progresar en la investigación sobre la importancia del factor empresarial en el sector turístico, atendiendo, en particular, al impacto económico de las pymes turísticas (Thomas et al., 2011; Russell y Faulkner, 2004). En general, las características de los empresarios condicionan el funcionamiento y los resultados de las pymes en cualquier sector y el impacto final de éstas, a nivel macroeconómico, sobre el desarrollo económico (Figura 1).

A nivel microeconómico, existen una serie de factores (organizacionales, económicos y del entorno) que determinan la aparición y la calidad de los emprendedores/empresarios, entendiendo que ésta se encuentra definida por su dinamismo emprendedor (Liñán y Chen, 2009; Guzmán y Rodríguez, 2008; Rodríguez y Santos, 2008; Santos y Liñán, 2007). La contribución de cada empresa a la generación de empleo, difusión de la innovación y crecimiento económico va a verse condicionada por la capacidad emprendedora del empresario, reflejada en su creatividad y capacidad de innovación, la tolerancia al riesgo e incertidumbre, su competencia para cooperar con otras empresas y agentes económicos, su actitud proactiva en la gestión empresarial, y sus aspiraciones de expansión y crecimiento para su empresa (Rodríguez, Moreno y Tejada, 2015; Romero, 2011). Esta capacidad emprendedora del empresario va a variar dependiendo de sus características personales, motivaciones y conocimientos (Guzmán y Santos, 2001).

Desde este punto de vista, la mayoría de las investigaciones realizadas sobre el papel del empresario y la empresa en la industria del turismo han llegado a la conclusión de que en este sector predominan los pequeños propietarios de negocios frente a los verdaderos empresarios emprendedores (Reijonen, 2008; Morrison et al., 1999), diferenciándose uno de otros en la escasa capacidad emprendedora de los primeros (Gilmore, Carson y O’Donell, 2004; Hankinson, Bartlett y Ducheneaut, 1997).

A su vez, a nivel mesoeconómico, la calidad de los emprendedores/empresarios condiciona las características de las empresas en un ámbito territorial determinado, es decir, la calidad del tejido empresarial (Romero, 2011; Fernández-Serrano y Romero, 2013). Ésta, por su parte, viene definida por una serie de factores que inciden en la capacidad de las empresas para impulsar el crecimiento y el desarrollo económicos en un territorio. Entre otros factores destacan la especialización sectorial de la empresa, sus esfuerzos en l+D, los encadenamientos productivos, la subcontratación, la cooperación, la internacionalización, o la innovación. De ellos dependen las externalidades originadas por la empresa, que a su vez, afectan a los resultados del resto de empresas del territorio.

Por último, a nivel macroeconómico, la calidad del tejido empresarial condiciona los resultados de las empresas en un ámbito espacial determinado y, en consecuencia, los resultados del territorio en términos de innovación, creación de empleo, crecimiento de la renta y, en definitiva, del desarrollo económico (Figura 1).

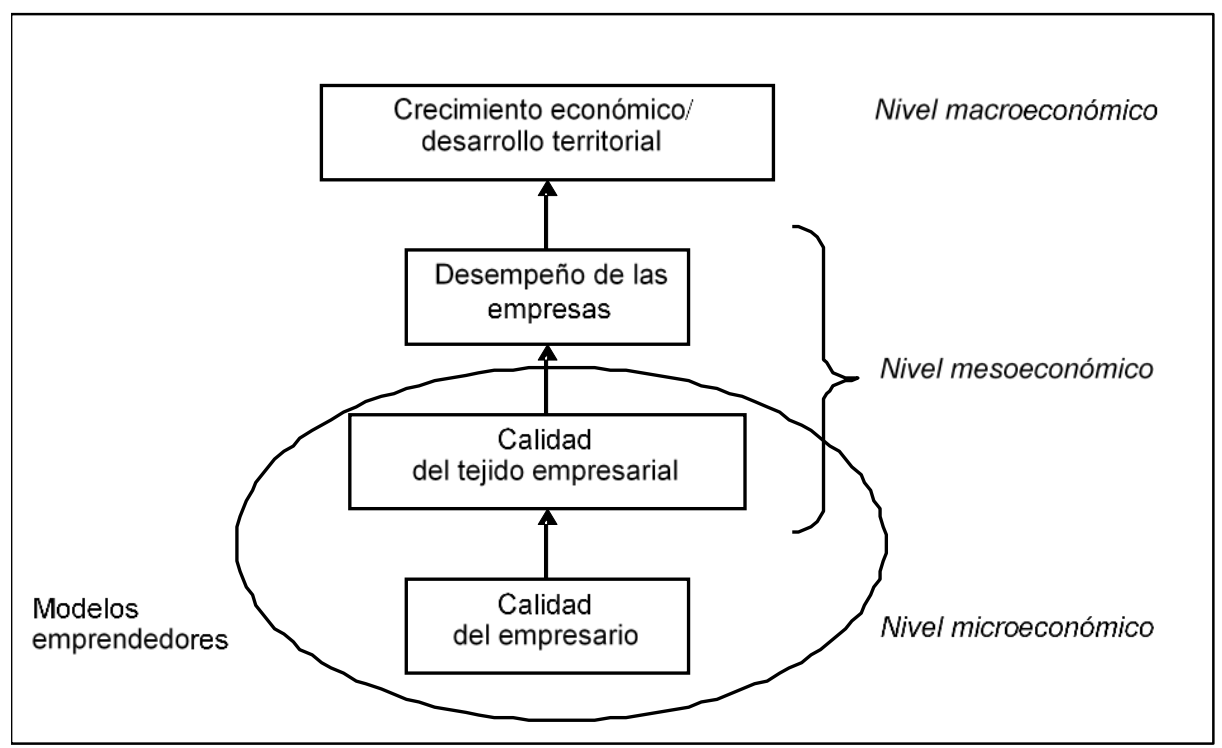

Figura 1: Calidad de los empresarios y el desarrollo territorial Fuente: Elaboración propia 


\section{METODOLOGÍA}

\subsection{Recolección de datos}

Para la elaboración de la parte empírica de este estudio se ha optado por la obtención de información primaria a través de una encuesta a pymes turísticas, dado que la información existente en fuentes estadísticas oficiales no permitía el estudio de buena parte de los aspectos de interés. La encuesta está dirigida a empresarios/gerentes de pymes en las siguientes actividades: alojamiento, servicios de comidas y bebidas y de agencias de viajes, operadores turísticos, servicios de reservas y actividades relacionadas con los mismos. El marco de la encuesta se obtuvo a partir de la información proporcionada por el DIRCE (INE, 2016). La definición operativa de pyme que se ha empleado en esta investigación es la de toda empresa que cuente al menos con un asalariado, pero que disponga de menos de 200 trabajadores, que hubiera tenido actividad en España a lo largo del año 2015.

Del total de la población objeto de estudio se seleccionó una muestra aleatoria mediante un procedimiento de muestreo estratificado con el objetivo de ajustar el tamaño por subsectores a nivel nacional. Aplicando un muestreo aleatorio simple, con un error del $5 \%$, una confianza del $90 \%$, considerando que la población es binomial $(P=Q=0,5$, situación más desfavorable) el tamaño de la muestra quedó determinado conforme se muestra en la Tabla 1, estratificándose por el tamaño de las empresas dentro de cada subsector turístico buscando la representatividad en cada estrato. El trabajo de campo se abordó a través de encuestas telefónicas asistidas por ordenador (CATI), a lo largo de los meses de Enero y Febrero de 2016.

El cuestionario completo consta de 130 ítems, combinando preguntas dicotómicas, categorizadas, de escala Likert y algunas de elección múltiple. Algunas preguntas del cuestionario se han adaptado de encuestas oficiales, como la "Encuesta sobre Innovación en las Empresas" del INE o la "Community Innovation Survey" de Eurostat, o de escalas de medida introducidas en investigaciones previas.

\begin{tabular}{|c|c|c|c|c|}
\hline & $\begin{array}{l}\text { Microempresas } \\
\text { (1-9 asalariados) }\end{array}$ & $\begin{array}{c}\text { Pequeñas empresas } \\
\text { (10-49 asalariados) }\end{array}$ & $\begin{array}{l}\text { Medianas empresas } \\
\text { (50-199 asalariados) }\end{array}$ & $\begin{array}{c}\text { Total } \\
\text { empresas }\end{array}$ \\
\hline Servicios de alojamiento (CNAE 55) & 179 & 135 & 61 & 375 \\
\hline $\begin{array}{l}\text { Servicios de comidas y bebidas } \\
\text { (CNAE 56) }\end{array}$ & 184 & 156 & 44 & 384 \\
\hline $\begin{array}{l}\text { Actividades de agencias de viajes, } \\
\text { operadores turísticos (CNAE 79) }\end{array}$ & 316 & 39 & 5 & 360 \\
\hline Total & 685 & 310 & 122 & 1.119 \\
\hline
\end{tabular}

Tabla 1: Muestra por subsectores y tamaño

Fuente: Elaboración propia a partir de datos del DIRCE (INE, 2016)

\subsection{Descripción de la muestra}

La muestra queda constituida por 1.119 pymes turísticas, siendo representativa de forma independiente para cada uno de los tres subsectores seleccionados. En el sector de alojamiento se han encuestado 375 establecimientos hoteleros, siendo el $61 \%$ de categorías superiores (tres, cuatro o cinco estrellas de oro), lo que se corresponde con la realidad de la población estudiada, es decir, la alta calidad de la industria hotelera española (Tabla 2). Pertenecientes al sector de la restauración se han encuestado 384 restaurantes o establecimientos de comidas, siendo el $50 \%$ de ellos de categorías medias, con precios que oscilan entre 15-35€ por servicio, y un $15,1 \%$ de gama alta, con precios superiores a $35 €$. Por último, en el sector de la intermediación turística se han encuestado 360 agencias de viajes, de las cuales un $75 \%$ son minoristas, un $20,3 \%$ mayoristasminoristas y solo un $4,7 \%$ son mayoristas (operadores turísticos).

\begin{tabular}{|l|l|l|l|l|l|}
\hline \multicolumn{2}{|c|}{ Categoría alojamiento } & \multicolumn{2}{c|}{ Categoría intermediación } & \multicolumn{2}{c|}{ Categoría restauración } \\
\hline Hostales & $11,2 \%$ & $\begin{array}{l}\text { Precios económicos y de } \\
\text { comida rápida }\end{array}$ & $34,1 \%$ & Minoristas & $75 \%$ \\
\hline Hotel 1 o 2 estrellas & $27,8 \%$ & Precios medios & $50 \%$ & Mayoristas-minoristas & $20,3 \%$ \\
\hline Hotel 3 o 4 estrellas & $54,9 \%$ & Gama alta & $15,1 \%$ & Mayoristas & $4,7 \%$ \\
\hline Hotel 5 estrellas & $6,1 \%$ & Gam &
\end{tabular}

Tabla 2: Categorías de los subsectores turísticos

Fuente: Elaboración propia

Respecto a la antigüedad de las empresas turísticas pertenecientes a la muestra, destacan las pymes más jóvenes en el sector de la intermediación restauración, al contrario que los hoteles, que presentan una mayor antigüedad (Tabla 3). Esto se relaciona con la menor supervivencia de las primeras, tal como se refleja en los datos de la Encuesta Demografía Armonizada de Empresas (INE, 2015). En cuanto a la dimensión empresarial de las pymes encuestadas, medida por el número de empleados, las empresas de mayor tamaño pertenecen al sector hotelero, con una media de 27 empleados. Por el contrario, el tejido empresarial de la intermediación turística es el que presenta un mayor grado de atomización empresarial. 
Uno de los principales retos del sector turístico español es alcanzar mayores niveles de calidad, siendo actualmente fundamental poseer algún distintivo o certificado de calidad específico del ámbito turístico, como la $Q$ de calidad turística. En la muestra estudiada, es el sector hotelero el que reúne un mayor número de pymes con dicho distintivo, aproximadamente una de cada tres. El sector de la intermediación registra los peores niveles. Por otro lado, actualmente se está viviendo un fenómeno de concentración hotelera, más acusado en las grandes empresas que en las pymes. Precisamente, el mayor número de pymes pertenecientes a cadenas 0 grupos empresariales se encuentra en el sector hotelero, en torno al $21 \%$ de las empresas encuestadas. Existe, por tanto, aún un amplio margen para avanzar en el proceso de concentración empresarial. Sin embargo, son las agencias de viajes las que más frecuentemente pertenecen a asociaciones de empresas independientes $(38,1 \%)$.

\begin{tabular}{|c|c|c|c|}
\hline Antigüedad de la empresa & Alojamiento & Intermediación & Restauración \\
\hline Edad media años (Desviación típica) & $22,93(16,21)$ & $17,45(11,26)$ & $18,55(15,85)$ \\
\hline$<10$ años & $14,4 \%$ & $26,7 \%$ & $24,0 \%$ \\
\hline $10-30$ & $65,6 \%$ & $61,1 \%$ & $72,7 \%$ \\
\hline$>30$ años & $20 \%$ & $12,2 \%$ & $3,4 \%$ \\
\hline \multicolumn{4}{|l|}{ Dimensión } \\
\hline № medio de empleados (Desviación típica) & $27,18(38,03)$ & $5,50(11,83)$ & $19,86(23,43)$ \\
\hline$[1-9]$ & $47,7 \%$ & $87,8 \%$ & $47,9 \%$ \\
\hline [10-49] & $36 \%$ & $10,8 \%$ & $40,6 \%$ \\
\hline [50-199] & $16,3 \%$ & $1,4 \%$ & $11,5 \%$ \\
\hline \multicolumn{4}{|l|}{ Distintivo de calidad turística } \\
\hline Sí & $34,4 \%$ & $18,1 \%$ & $31,5 \%$ \\
\hline No & $65,6 \%$ & $81,9 \%$ & $68,5 \%$ \\
\hline \multicolumn{4}{|l|}{ Pertenencia grupo de empresa } \\
\hline Sí & $21,6 \%$ & $19,2 \%$ & $13,5 \%$ \\
\hline No & $78,4 \%$ & $80,0 \%$ & $86,5 \%$ \\
\hline \multicolumn{4}{|c|}{ Pertenencia cadena hotelera/de restaurantes/de agencias } \\
\hline Sí & $21,3 \%$ & $19,2 \%$ & $17,2 \%$ \\
\hline No & $78,7 \%$ & $80,8 \%$ & $82,8 \%$ \\
\hline \multicolumn{4}{|c|}{ Pertenencia asociación de empresas independientes } \\
\hline Sí & $24,5 \%$ & $38,1 \%$ & $30,2 \%$ \\
\hline No & $75,5 \%$ & $61,9 \%$ & $69,8 \%$ \\
\hline \multicolumn{4}{|c|}{ Pertenencia una red de comercialización o distribución } \\
\hline Sí & $13,6 \%$ & - & $13,8 \%$ \\
\hline No & $86,4 \%$ & - & $86,2 \%$ \\
\hline
\end{tabular}

Tabla 3: Características comunes de los subsectores turísticos de la muestra Fuente: Elaboración propia

\section{PERFIL DEL EMPRESARIO TURÍSTICO}

Como se ha señalado previamente, la creación de riqueza y el dinamismo de una economía se sustenta sobre la competitividad de sus empresas, y ésta depende de las capacidades de sus empresarios, que viene determinada por sus características personales (Russell y Faulkner, 2004).

\subsection{Perfil demográfico de los empresarios}

Atendiendo a los resultados de nuestro estudio se observa que la mayor parte de los empresarios encuestados son varones de edades media-avanzada, en torno a los 45 años, no inmigrantes, y con niveles de estudios medio-superiores. Estos empresarios cuentan con una amplia experiencia laboral, especialmente por cuenta ajena y en actividades turísticas (Tabla 4).

En general, los resultados relativos al género y la edad coinciden con los de estudios previos realizados en el sector (Jaafar, Abdul-Aziz, Maideen y Mohd, 2011, Ahmad, 2005; Morrison, Breen, y Ali, 2003; Szivas, 2001; Getz y Carlsen, 2000). No obstante, en relación al género del empresario, se observan algunas diferencias interesantes entre subsectores. Así, aunque las mujeres representan una pequeña proporción del total de empresarios de la muestra, especialmente en el sector de la restauración, su presencia es algo mayor en el sector de las agencias de viajes y operadores turísticos. Esto contradice los resultados obtenidos en estudios previos que indican que las empresas gestionadas por mujeres tienden a concentrarse en actividades turísticas que son una extensión de sus tradicionales roles domésticos (Apostolopoulos y Sönmez, 2001; Cohen 2001), como, por ejemplo, pequeños negocios familiares de hospedaje o de restauración localizados en el entorno del hogar (Teo y Lim, 2003; Hall 2001).

Por lo que se refiere al nivel educativo de los empresarios turísticos, los resultados de estudios previos son inconsistentes. En algunos trabajos, los empresarios con estudios superiores representan una parte pequeña del total (Sharma y Upneja, 2005; Getz y Carlsen, 2000), en otros, por el contrario, son mayoría los empresarios que 
cuentan con estudios superiores (Szivas, 2001). En el presente trabajo, los empresarios del subsector de hotelería e intermediación poseen mayoritariamente estudios superiores. Por el contrario, en el sector de la restauración son más numerosos los que tienen estudios medios. Lo cual confirmaría las carencias tradicionales en la formación del personal de restauración (García, Campos, Sánchez y Marchante, 2011). Por el contrario, los niveles de formación más altos se encuentran en los empresarios del subsector de la intermediación turística. Con relación a esta cuestión, se ha argumentado que algunos empresarios del sector de la hostelería consideran no necesitar formación específica porque ya conocen las particularidades de sus negocios, mientras que otros sí reconocen tener lagunas de conocimiento (Dewhurst, Dewhurst y Livesey, 2007), aunque señalan que esta deficiencia no les incapacita para gestionar sus negocios (Schroeder, 2003). Ello explicaría la baja participación de estos empresarios en cursos de formación especializada relacionados con el negocio del turismo (Schroeder, 2003).

Respecto a la experiencia laboral, también los resultados obtenidos coinciden con los de estudios previos, en los que se señala que debido a las bajas barreras de entrada a esta industria, los empresarios cuentan con una amplia experiencia en diversas ocupaciones antes de iniciarse en la actividad empresarial en el sector (Szivas, 2001; Ateljevic, Milne, Doorne y Ateljevic, 1999). No obstante, en el actual estudio se observa que, salvo en el subsector de la intermediación, la experiencia específica en actividades turísticas, tanto por cuenta ajena como por cuenta propia en otro negocio anterior, no es habitual entre los empresarios encuestados. Así pues, solo uno de cada tres empresarios de los subsectores de alojamiento y restauración acreditaba experiencia previa como trabajador por cuenta ajena en el sector turístico antes de iniciar su actividad empresarial actual. De la misma forma, tampoco son muy numerosos los empresarios con experiencia previa por cuenta propia en otro negocio en el propio sector turístico o en otras actividades (en torno a un tercio de los encuestados).

\begin{tabular}{|c|c|c|c|}
\hline Descripción & Alojamiento & Intermediación & Restauración \\
\hline \multicolumn{4}{|l|}{ Género } \\
\hline Varones & $65,6 \%$ & $58,3 \%$ & $76,0 \%$ \\
\hline Mujeres & $34,4 \%$ & $41,7 \%$ & $24,0 \%$ \\
\hline \multicolumn{4}{|l|}{ Edad Media } \\
\hline № medio de años/Desviación típica & $46,78(11,37)$ & $47,5(9,39)$ & $47,2(10,13)$ \\
\hline Menos de 30 años & $4,5 \%$ & $2,2 \%$ & $3,4 \%$ \\
\hline Entre 30 y 50 años & $59,5 \%$ & $60,6 \%$ & $59,4 \%$ \\
\hline Más de 50 años & $35,2 \%$ & $36,7 \%$ & $36,7 \%$ \\
\hline \multicolumn{4}{|l|}{ Inmigrante } \\
\hline Sí & $14,7 \%$ & $7,5 \%$ & $9,9 \%$ \\
\hline No & $85,3 \%$ & $92,5 \%$ & $90,1 \%$ \\
\hline \multicolumn{4}{|l|}{ Nivel de educación } \\
\hline Sin estudios & $1,6 \%$ & - & $1,3 \%$ \\
\hline$E G B$, primaria o secundaria & $8,3 \%$ & $2,8 \%$ & $17,2 \%$ \\
\hline$F P$, bachillerato, $B U P$ & $25,1 \%$ & $25,3 \%$ & $43,9 \%$ \\
\hline Estudios superiores & $65,1 \%$ & $71,9 \%$ & $38,5 \%$ \\
\hline \multicolumn{4}{|l|}{ Experiencia Laboral } \\
\hline \multicolumn{4}{|l|}{ Por cuenta ajena } \\
\hline Sí & $60,8 \%$ & $75 \%$ & $66,4 \%$ \\
\hline En actividad turística & $37,1 \%$ & $52,5 \%$ & $39,3 \%$ \\
\hline En actividad no turística & $23,7 \%$ & $22,5 \%$ & $27,1 \%$ \\
\hline No & $39,2 \%$ & $25 \%$ & $33,6 \%$ \\
\hline \multicolumn{4}{|l|}{ Por cuenta propia } \\
\hline Sí & $33,0 \%$ & 27,2 & $38,0 \%$ \\
\hline En actividad turística & $16,5 \%$ & $15 \%$ & $22,4 \%$ \\
\hline En actividad no turística & $16,5 \%$ & $12,2 \%$ & $15,6 \%$ \\
\hline No & $66,9 \%$ & $72,8 \%$ & $62,0 \%$ \\
\hline
\end{tabular}

Tabla 4: Características del empresario turístico Fuente: Elaboración propia

Algunos investigadores de la teoría del empresario consideran que a través de la educación y experiencia laboral el empresario puede adquirir capacidades y aptitudes útiles para desarrollar una actividad empresarial (Wiklund y Shepherd, 2005; Storey, 2000; Ray, 1993; Stanworth y Gray, 1991; Cooper y Dunkelberg, 1987; Schultz, 1985), lo que nos permite suponer a priori, dado el elevado nivel de formación y la experiencia laboral de los empresarios de turismo seleccionados para este estudio, que poseen estas capacidades en cierta medida. Sin embargo, otros estudios en este campo señalan que no es suficiente una formación estándar, sino que se requiere una formación especializada dirigida explícitamente tanto a desarrollar las habilidades y destrezas de gestión empresarial, como las capacidades y competencias emprendedoras (Guzmán y Cáceres, 2001; Gibb, 1994). Como se ha visto al analizar el perfil del empresario turístico, los empresarios encuestados no parecen poseer esa experiencia laboral específica que, bien porque se haya adquirido en actividades de turismo, o bien porque se haya adquirido desarrollando una actividad empresarial por cuenta propia, les haya permitido adquirir los conocimientos específicos que les permitan desarrollar actitudes, capacidades y comportamientos emprendedores. 


\subsection{Motivaciones de los empresarios turísticos}

La inclinación de los individuos al desarrollo de una actividad empresarial, y la eficiencia y dinamismo con la que posteriormente desempeñen la gestión de la misma, va a depender, entre otros determinantes, de sus motivaciones (Davidsson, 1991). Estas motivaciones, relacionadas con los intereses personales de los empresarios, condicionan sus actitudes emprendedoras (Krueger, 2003). El empresario valorará los rendimientos de todo tipo, intrínsecos (satisfacción, realización, independencia...), extrínsecos (poder, dinero, reconocimiento, empleo...), o transcendentales (ayuda a los demás, cuidado de los hijos, etc.), que espera obtener si se decide a iniciar un nuevo negocio, o adopta ciertos comportamientos más o menos emprendedores. Cuando los resultados y las consecuencias de esos comportamientos coinciden con los intereses del empresario, éste se verá motivado a adoptar esas conductas y decisiones (Guzmán y Santos, 2001).

Entre los individuos encuestados para este estudio, son las motivaciones por oportunidad, como el desarrollo personal y profesional, el deseo de independencia y autonomía profesional y las oportunidades de negocio en el mercado turístico, las que tienen más peso en su decisión de convertirse en empresarios turísticos (Tabla 5). Se considera generalmente que estos tipos de motivación (frente a la motivación por necesidad) atraen hacia los negocios a los empresarios con mejores capacidades y competencias emprendedoras, quienes suelen tener unas actitudes más positivas hacia el crecimiento de sus empresas y tienden a adoptar comportamientos más dinámicos en su gestión (Peña, Guerrero y González-Pernía, 2016; Beaver, 2002).

\begin{tabular}{|l|r|r|r|}
\hline Motivación & Alojamiento & Intermediación & Restauración \\
\hline (1 total desacuerdo, 7 total acuerdo) & $\begin{array}{c}\text { Media } \\
\text { (Desv. típica) }\end{array}$ & $\begin{array}{c}\text { Media } \\
\text { (Desv. típica) }\end{array}$ & $\begin{array}{c}\text { Media } \\
\text { (Desv. típica) }\end{array}$ \\
\hline Oportunidad & & \multicolumn{3}{|c|}{} \\
\hline Desarrollo personal y profesional & $5,19(1,988)$ & $5,60(1,931)$ & $5,29(2,063)$ \\
\hline Oportunidad negocio en mercado turístico & $4,82(1,956)$ & $2,12(2,177)$ & $4,46(2,253)$ \\
\hline Independencia & $4,63(2,209)$ & $5,36(2,096)$ & $4,99(2,242)$ \\
\hline Ingresos elevados & $4,52(2,046)$ & $4,32(2,127)$ & $4,46(2,182)$ \\
\hline Necesidad & & & \\
\hline Continuar negocio familiar & $4,20(2,777)$ & $4,20(2,263)$ & $3,72(2,812)$ \\
\hline Desempleo, precariedad laboral & $2,60(2,079)$ & $2,52(2,164)$ & $2,44(2,162)$ \\
\hline
\end{tabular}

Tabla 5: Motivaciones empresariales

Fuente: Elaboración propia

Los resultados obtenidos coinciden con otros estudios, que muestran que los empresarios del sector de turismo se ven atraídos hacia la actividad empresarial por el deseo de desarrollar un estilo de vida asociado a las actividades turísticas (Lashley y Rowson, 2009; Mottiar, 2007; Getz y Carlsen, 2000; Hall y Rusher, 2004; Szivas, 2001), el deseo de trabajar por cuenta propia y la detención de una oportunidad de negocio (Bransgrove y King, 1996). Estos resultados contradicen una percepción ampliamente extendida sobre la puesta en marcha de negocios en el sector turístico como salida forzada frente a los altos niveles de desempleo en España.

Aunque en los tres subsectores estudiados el deseo de desarrollo personal y profesional es la principal motivación empresarial, se observan algunas diferencias entre ellos. Así, en el sector de la hostelería la percepción de una oportunidad de negocio y el deseo de explotarla a través del desarrollo de una actividad empresarial se convierte en la segunda fuente de motivación empresarial. Por el contrario, en los subsectores de agencias de viajes y de restauración, se da prioridad a la autonomía e independencia profesional y al deseo de obtener ingresos elevados. Esto último contradice las conclusiones obtenidas en otros estudios sobre las motivaciones de los empresarios en la industria del turismo, en los que se establece que el deseo de maximizar beneficios no es una motivación frecuente entre los empresarios del sector (Dewhurst y Horobin, 1998), que solo perseguirían una ganancia financiera a largo plazo (Bransgrove y King, 1996).

\subsection{Actitudes hacia el crecimiento del empresario turístico}

Las motivaciones de los empresarios van a condicionar sus actitudes respecto a la evolución futura de sus empresas. En general, las investigaciones realizadas sobre el empresario en el ámbito del turismo señalan que, aunque algunos empresarios turísticos buscan el crecimiento de sus negocios, la mayoría limitan el tamaño de su empresa a un nivel que les garantice cierta calidad de vida compatible con el desarrollo de esta actividad profesional (Thomas, Shaw y Page, 2011). Así, Shaw (2003) distingue entre empresarios emprendedores orientados al negocio, y pequeños propietarios de negocios asociados a un estilo de vida relacionado con la actividad turística que desempeñan.

En el caso de nuestro estudio, se observa que las actitudes de los empresarios respecto al crecimiento de sus empresas muestran una ambición moderada, en el sentido de que no parecen desear una empresa de gran tamaño (Tabla 6). Coinciden así los resultados de este trabajo con otras investigaciones previas. Por subsectores, no se advierten diferencias apreciables en las actitudes hacia el crecimiento de los empresarios. 


\begin{tabular}{|c|c|c|c|}
\hline & Alojamiento & Intermediación & Restauración \\
\hline & $\begin{array}{c}\text { Media } \\
\text { (Desv. típica) }\end{array}$ & $\begin{array}{c}\text { Media } \\
\text { (Desv. típica) }\end{array}$ & $\begin{array}{c}\text { Media } \\
\text { (Desv.típica) }\end{array}$ \\
\hline \multicolumn{4}{|l|}{ Tamaño deseado de la empresa en un futuro } \\
\hline (1 Manejable, con pocos empleados, 7 lo más grande posible) & $\begin{array}{c}4,82 \\
(2,244)\end{array}$ & $\begin{array}{c}4,42 \\
(2,469)\end{array}$ & $\begin{array}{c}4,99 \\
(2,213)\end{array}$ \\
\hline \multicolumn{4}{|l|}{ Un crecimiento $100 \%$ de empleados en 5 años } \\
\hline (1 muy negativo, 7 muy positivo) & $\begin{array}{c}5,06 \\
(1,971)\end{array}$ & $\begin{array}{c}5,37 \\
(1,949)\end{array}$ & $\begin{array}{c}5,29 \\
(2,002)\end{array}$ \\
\hline \multicolumn{4}{|l|}{ Un crecimiento $100 \%$ de ventas en 5 años } \\
\hline (1 muy negativo, 7 muy positivo) & $\begin{array}{c}6,19 \\
(1,405)\end{array}$ & $\begin{array}{c}6,00 \\
(1,588)\end{array}$ & $\begin{array}{c}6,03 \\
(1,539) \\
\end{array}$ \\
\hline
\end{tabular}

Tabla 6: Actitudes hacia el crecimiento Fuente: Elaboración propia

\subsection{Orientación emprendedora del empresario turístico.}

Las actitudes del empresario sobre la evolución futura de su empresa van a condicionar los comportamientos que adopte para su dinamización. Las empresas con una clara orientación emprendedora, que son las que mayor contribución realizan a la construcción de un tejido empresarial de calidad y al crecimiento económico de un territorio, reflejan unas actitudes ambiciosas respecto al crecimiento en sus empresarios, así como las capacidades y competencias de los mismos para alcanzar los objetivos deseados (Madsen, 2007; Wiklund y Shepherd, 2005). Los empresarios más ambiciosos y emprendedores suelen ser más innovadores y creativos en el desarrollo de la actividad empresarial, más tolerantes a la incertidumbre, capaces de asumir riesgos moderados en sus operaciones empresariales, y más proactivos a la hora de actuar y gestionar su empresa (Lumpkin y Dess, 1996; Davidsson, 1991).

Los resultados obtenidos indican una reducida orientación emprendedora en la empresa turística en España (Tabla 7). Este resultado coincide con los de otras investigaciones sobre la orientación emprendedora de las pymes turísticas que establecen que ésta parece ser escasa, especialmente respecto a la innovación (Morrison, 2006; Getz y Petersen, 2005; Page et al., 1999; Thomas et al., 1998).

\begin{tabular}{|c|c|c|c|}
\hline & Alojamiento & Intermediación & Restauración \\
\hline (7 totalmente de acuerdo a 1 total desacuerdo) & $\begin{array}{c}\text { Media } \\
\text { (Desv. típica) }\end{array}$ & $\begin{array}{c}\text { Media } \\
\text { (Desv. típica) } \\
\end{array}$ & $\begin{array}{c}\text { Media } \\
\text { (Desv. típica) }\end{array}$ \\
\hline $\begin{array}{l}\text { Orientación Emprendedora } \\
\text { (media Innovación, Riesgo y Proactividad) }\end{array}$ & $3,92(1,38)$ & $3,82(1,35)$ & $4,04(1,483)$ \\
\hline Innovación & $\mathbf{4 , 2 7}(1,63)$ & $\mathbf{4 , 1 9}(1,638)$ & $\mathbf{4 , 2 0}(1,789)$ \\
\hline $\begin{array}{l}\text { Mi empresa da gran importancia a las actividades de } I+D, \\
\text { a la innovación y al liderazgo tecnológico. }\end{array}$ & $4,62(1,749)$ & $4,63(1,845)$ & $4,52(2,004)$ \\
\hline $\begin{array}{l}\text { Mi empresa ha comercializado nuevas líneas de productos } \\
\text { o servicios en los últimos } 5 \text { años }\end{array}$ & $4,36(2.057)$ & $\begin{array}{c}4,55 \\
(2,143)\end{array}$ & $4,42(2,208)$ \\
\hline $\begin{array}{l}\text { Mi empresa ha introducido cambios radicales en sus } \\
\text { líneas de productos o servicios en los últimos } 5 \text { años }\end{array}$ & $3,84(2,069)$ & $3,44(2,097)$ & $3,66(2,193)$ \\
\hline Riesgo & $3,46(1,59)$ & $3,60(1,503)$ & $3,71(1,661)$ \\
\hline $\begin{array}{l}\text { En mi empresa se es muy proclive a proyectos de alto } \\
\text { riesgo (proyectos capaces de obtener rentabilidades muy } \\
\text { altas) }\end{array}$ & $2,58(1,820)$ & $2,41(1,717)$ & $2,72(1,947)$ \\
\hline $\begin{array}{l}\text { En mi empresa se cree que debido a la naturaleza del } \\
\text { entorno se necesitan actos valientes y de gran alcance } \\
\text { para lograr los objetivos de la empresa }\end{array}$ & $3,75(1,998)$ & $4,04(1,966)$ & $3,92(2,131)$ \\
\hline $\begin{array}{l}\text { En mi empresa cuando hay que tomar decisiones en } \\
\text { situaciones de incertidumbre, se suele adoptar una } \\
\text { postura valiente y agresiva para aprovechar al máximo las } \\
\text { oportunidades }\end{array}$ & $4,07(1,924)$ & $4,37(1,973)$ & $4,50(1,97)$ \\
\hline Proactividad & $\mathbf{4 , 0 2}(1,75)$ & $3,82(1,754)$ & $\mathbf{4 , 2 2}(1,483)$ \\
\hline $\begin{array}{l}\text { En las relaciones con la competencia, es mi empresa la } \\
\text { que normalmente inicia acciones a las que responden los } \\
\text { competidores }\end{array}$ & $4,03(1,863)$ & $3,74(1,903)$ & $4,10(2,034)$ \\
\hline $\begin{array}{l}\text { Mi empresa es a menudo la primera en introducir nuevos } \\
\text { productos, servicios, técnicas de gestión, etc., antes que } \\
\text { el resto de competidores }\end{array}$ & $3,94(1,911)$ & $3,72(1,904)$ & $4,22(2,062)$ \\
\hline $\begin{array}{l}\text { En general en mi empresa se tiene una fuerte tendencia a } \\
\text { ir por delante de otros competidores en la introducción de } \\
\text { nuevos productos e ideas }\end{array}$ & $4,09(1,94)$ & $3,99(1,880)$ & $4,33(2,068)$ \\
\hline
\end{tabular}

Tabla 7: Percepción del empresario sobre la orientación emprendedora de su empresa Fuente: Elaboración propia 
No obstante lo anterior, examinando los diferentes componentes de la orientación emprendedora en la muestra correspondiente a este estudio se aprecia que es la orientación hacia la innovación la que parece tener más desarrollada los empresarios, con independencia del sector de actividad turística considerado (Tabla 7). Por el contrario, la tolerancia a la incertidumbre y la capacidad de tomar decisiones que impliquen asumir un cierto nivel de riesgo no caracteriza a los empresarios del turístico encuestados en este estudio, como tampoco su actitud proactiva en la gestión empresarial. Esto podría deberse a las características intrínsecas de cada uno de estos subsectores, puesto que hay indicios en las investigaciones previas realizadas sobre este tema de una mayor incidencia de la innovación entre las empresas que operan en estos tres sectores, y de una menor orientación emprendedora en las empresas pequeñas de carácter familiar (Getz y Petersen, 2005).

\section{CONCLUSIONES}

Este estudio descriptivo pone de relieve algunas de las principales características de los empresarios de las pymes en un sector como el turístico, que es clave para la economía española.

Los resultados obtenidos contribuyen a desarrollar un área de investigación que ha sido calificada como emergente e incompleta (Page et al., 1999), y en la que es necesario avanzar tanto desde la perspectiva teórica como empírica (Thomas et al., 2011). Con este trabajo se pretende contribuir a un mejor conocimiento acerca de los procesos empresariales en el turismo y su impacto económico, el papel que juega el empresario turístico y, las similitudes o diferencias en los resultados de las pymes y características de los empresarios en los diferentes subsectores turísticos. Al mismo tiempo, se ofrecen conclusiones que superan las limitaciones de estudios previos referidos a ámbitos geográficos y actividades turísticas muy concretas.

Los resultados apuntan a una moderada capacidad emprendedora de los empresarios del sector en España, lo que repercute en el desempeño de sus empresas y, por extensión, en la calidad del tejido empresarial turístico. Parece que en el sector predominan los pequeños propietarios frente a los empresarios emprendedores, lo que está relacionado con las motivaciones que atraen a los empresarios turísticos hacia la actividad empresarial, así como con sus actitudes respecto al crecimiento de las empresas.

Se aprecia en estos empresarios una percepción del éxito empresarial que no se corresponde a menudo con criterios estrictamente económicos, como las ganancias o el tamaño de la empresa, sino más bien con el deseo de compaginar sus objetivos personales (calidad de vida, servicio a la comunidad, un estilo de vida que les permite disfrutar del entorno) con sus objetivos empresariales (satisfacer a clientes y empleados, reforzar la imagen de su marca turística, rentabilizar el negocio).

El estudio realizado ha quedado limitado a un análisis meramente descriptivo, por lo que queda pendiente para futuras investigaciones profundizar en los resultados encontrados utilizando técnicas estadísticas que permitan aclarar el sentido de las relaciones encontradas.

\section{BIBLIOGRAFÍA}

Ahmad, G. (2005). Small firm owner-managers networks in tourism and hospitality. International Journal of Business and Society, 4(2), 37-55.

Andriotis, K. (2002). Scale of hospitality firms and local economic development e evidence from Crete. Tourism Management, 23(4), 333-341. doi:10.1016/S0261-5177(01)00094-2

Apostolopoulos, Y., y Sonmez, S. (2001). Working producers, leisured consumers: Women's experiences in developing regions. En: Y. Apostolopoulos, S. Sonmez y D. J. Timothy (Eds), Women as Producers and Consumers of Tourism in Developing Regions (pp. 3-18). Westport CT, USA: Praeger Publishers.

Ateljevic, J. y Li, L. (2009). Tourism entrepreneurship: Concepts and issues. En: J. Ateljevic and P. Stephen (Eds), Tourism and Entrepreneurship: International Perspectives (pp. 9-32). Oxford, UK: Butterworth Heinemann.

Ateljevic, J., Milne, S., Doorne, S. y Ateljevic, I. (1999). Tourism Micro-firms in Rural New Zealand Key Issues for the Coming Millennium. Victoria University Tourism Research Group. Wellington, New Zealand.

Beaver, G. (2002). Strategy and management in the smaller enterprise. Journal of Strategic Change, 11, 175-181. doi:10.1002/jsc.591.

Bransgrove, C. y King, B. (1996). Strategic marketing practice amongst small tourism and hospitality businesses. En: Thomas, R. (Ed.), Spring Symposium Proceedings of International Association of Hotel Management Schools (29-38). Leeds, UK: Leeds Metropolitan University.

Brida, J.G., Pereyra, J.S., Such, M.J. y Zapata, S. (2008). La contribución del turismo al crecimiento económico. Cuadernos de turismo, 22, 35-46. 
Cohen, C. B. (2001). Island is a woman: women as producers and products in British Virgin Islands tourism. En: Y. Apostolopoulos, S. Sonmez, y D. J. Timothy (Eds.), Women as Producers and Consumers of Tourism in Developing Regions (47-72). Westport CT, USA: Praeger Publishers.

Cooper, A.C. y Dunkelberg, W.C. (1987). Entrepreneurial Research: Old Questions, New Answers, and Methodological Issues. American Journal of Small Business, 3, 11-23.

Cuadrado, J.R. y López, J.M. (2014). El turismo, motor del crecimiento y de la recuperación de la economía española. Revista de Estudios Turísticos, 200, 19-34.

Davidsson, P. (1991). Continued entrepreneurship: ability, need, and opportunity as determinants of small firm growth. Journal of Business Venturing, 6, 405-429.

Dewhurst, H., Dewhurst, P. y Livesey, R. (2007). Tourism and hospitality SME training needs and provision: a sub-regional analysis. Tourism and Hospitality Research, 7(2), 131-143.

Dewhurst, P. y Horobin, H. (1998). Small business owners. En R. Thomas (Ed.), The management of small tourism and hospitality firms. London, UK: Cassell.

Fuller, D., Buultjens, J. y Cummings, E. (2005). Ecotourism and indigenous microenterprise formation in northern Australia e opportunities and constraints. Tourism Management, 26(6), 891-904. doi: 10.1016/j.tourman.2004.04.006

García, A., Campos, J.A., Sánchez, J.L. y Marchante, M. (2011). Capital humano y salarios en la hostelería española: un análisis regional. Revista de Estudios Regionales, 91, 71-96.

Getz, D. y Carlsen, J. (2000). Characteristics and goals of family and owner-operated business in the rural tourism and hospitality sectors. Tourism Management, 21, 547-560.

Getz, D y Petersen, T (2005). Growth and profit-oriented entrepreneurship among family business owners in the tourism and hospitality industry International, Journal of Hospitality Management, 24(2), 219-242. doi: 10.1016/j.ijhm.2004.06.007

Gibb, A.A. (1994). Do we really teach small business the way we should. Journal of Small Business and Entrepreneurship, 11(2), 11-28. doi:10.1080/08276331.1994.10600453

Gilmore, A., Carson, D. y O'Donnell, A. (2004). ENTREPRENEURS AT THE INTERFACE AND THEIR ATTITUDE TO RISK. Marketing Intelligence and Planning, 22(3), $349-360$. doi:10.1108/02634500410536920

Guzmán, J. y Cáceres, R. (2001): Un modelo explicativo de la emergencia de empresarios. Economía Industrial, 340, 151-162.

Guzmán, J. y Santos, F.J. (2001). The Booster Function and the Entrepreneurial Quality: An Application to the Province of Seville. Entrepreneurship and Regional Development, 13(3), 211-228. doi:10.1080/08985620110035651

Guzmán, J. y Rodríguez, M.J. (2008). Comportamiento de las mujeres empresarias: una visión global. Revista de Economía Mundial, 18, 367- 378.

Hall, C. M. y Rusher, K. (2004). Risky lifestyles? Entrepreneurial characteristics of the New Zealand bed and breakfast sector. En R. Thomas (Ed.), Small firms in tourism: International perspectives (pp 83-98). Amsterdam, Netherlands: Elsevier:

Hall, D.R. (2001). From the iron curtain to the dollar curtain: Women and tourism in Eastern Europe. En Y. Apostolopoulos, S. Sonmez, y D. J. Timothy (Eds.), Women as Producers and Consumers of Tourism in Developing Regions (pp 191-210). Westport CT, USA: Praeger Publishers.

Hankinson, A., Bartlett, D. y Ducheneaut, B. (1997). The Key Factors in the Small Profiles of Small-Medium Enterprise Owner-Managers that Influence Business Performance: The UK (Rennes) SME Survey 19951997 An International Research Project UK survey. International Journal of Entrepreneurial Behavior and Research, 3(4), 68-82.

INE (2015). Demografía Armonizada de Empresas. Consultado 09/05/16: http://www.ine.es/jaxi/menu.do?type=pcaxis\&path=\%2Ft37\%2Fp204\&file=inebase\&L=0

INE (2016). Directorio Central de Empresas. Consultado 09/05/16: http://www.ine.es/jaxi/menu.do?type=pcaxis\&path=\%2Ft37\%2Fp201\&file=inebase \&L=0

Jaafar, M., Abdul-Aziz, A.R., Maideen, S.A. y Mohd, S.Z. (2011). Entrepreneurship in the tourism industry: Issues in developing countries. International Journal of Hospitality Management, 30, 827-835. doi: 10.1016/j.jhm.2011.01.003

Krueger, N.F. (2003). The cognitive psychology of entrepreneurship. En Z. Acs y D.B. Audretsch (Eds.), Handbook of Entrepreneurial Research (pp 105-140). London, UK: Kluwer Law International. 
Lashley, C., y Rowson, B. (2009). Lifestyle businesses: insights into Blackpool's hotel sector. International Journal of Hospitality Management, 29(3), 511-519. doi: 10.1016/j.jihm.2009.10.027

Liñán, F. y Chen, Y.W. (2009). Development and Cross-Cultural application of a specific instrument to measure entrepreneurial intentions. Entrepreneurship Theory and Practice, 33(3), 593-617. doi:10.1111/j.15406520.2009.00318.x

Lumpkin, G.T. y Dess, G.G. (1996). Clarifying the entrepreneurial orientation construct and linking it to performance. Academy of Management Review, 21, 135-172. doi:10.5465/AMR.1996.9602161568

Madsen, E.L. (2007). The Significance of Sustained Entrepreneurial Orientation on Performance of Firms - A Longitudinal Analysis. Entrepreneurship and Regional Development, 19(2), 185-204. doi:10.1080/08985620601136812

Morrison, A. (2003). Conference review: international conference e small firms in the tourism and hospitality sectors. Tourism Management, 24(4), 495-498. doi:10.1016/S0261-5177(02)00118-8

Morrison, A. (2006). A contextualisation of entrepreneurship. International Journal of Entrepreneurial Behaviour and Research, 12(4), 192-209. doi:10.1108/13552550610679159

Morrison, A., Breen, J. y Ali, S. (2003). Small business growth: intention, ability and opportunity. Journal of Small Business Management, 41(4), 417-425. doi:10.1111/1540-627X.00092

Morrison, A., Rimmmingtin, M. y Williams, C. (1999). Entrepreneurship in the Hospitality, Tourism and leisure industries. Oxford, UK: Butterworth \& Heinemann.

Mottiar, Z. (2007). Lifestyle entrepreneurs and spheres of inter-firm relations: the case of Westport, Co mayo, Ireland. International Journal of Entrepreneurship and Innovation, 8(1), 67-74. doi:10.5367/000000007780007326

Novelli, M., Schmitz, B. y Spencer, T. (2005). Networks, clusters and innovation in tourism: a UK experience. Tourism Management, 27(6), 1141-1152. doi: 10.1016/j.tourman.2005.11.011

Organisation for Economic Cooperation and Development (2008). OECD competitiveness and sustainable global tourism markets. In Briefing paper for the high level ministerial tourism committee meeting. Riva de la Garde, Italy.

OMT (2016). "Las exportaciones del turismo internacional crecen un 4\% en 2015”. Nota de Prensa 06/05/2016.

Page, S.J., Forer, P. y Lawton, G.R. (1999). Small business development and tourism: terra incognita? Tourism Management, 20(4), 435-459. doi:10.1016/S0261-5177(99)00024-2

Peña, I., Guerrero, M. y González-Pernía, J.L. (2016). Informe GEM España 2015. Santander, España: Editorial de la Universidad de Cantabria.

Ray, D.M. (1993). Understanding the entrepreneur: Attributes, Experiences and Skill. Entrepreneurship and Regional Development, 5(4), 345-357. doi:10.1080/08985629300000022

Reichel, A., y Haber, S. (2005). A three-sector comparison of the business performance of small tourism enterprises: an exploratory study. Tourism Management., 26(5), 681-690. doi: 10.1016/j.tourman.2004.03.017

Reijonen, H. (2008). Understanding the small business owner: what they really aim at and how this relates to firm performance: A case study in North Karelia, Eastern Finland. Management Research News. 31(8), 616629. doi:10.1108/01409170810892172

Rodríguez, M.J. y Santos, F.J. (2008). Women Nascent Entrepreneurs and Social Capital in the Process of Firm Creation. THE INTERNATIONAL ENTREPRENEURSHIP AND MANAGEMENT JOURNAL. vol. 4(2), 1-50. doi:10.1007/s11365-007-0070-z

Rodríguez, M.J., Moreno, P. y Tejada, P. (2015). La contribución de las pymes turísticas al desarrollo económico. Revista de Economía Mundial, 35,101-117.

Romero, I. (2011). Analysing the composition of the SME sector in high and low - income regions: Some research hypotheses. Entrepreneurship and Regional Development, 23(7-8), 637-660. doi:10.1080/08985626.2010.491872

Fernández-Serrano, J. y Romero, I. (2013). Entrepreneurial quality and regional development: Characterising SME sectors in low income areas. Papers in Regional Science, 92 (3), 495-513. doi:10.1111/j.14355957.2012.00421.X

Russell, R. y Faulkner, B. (2004). Entrepreneurship, Chaos and the Tourism Area Lifecycle. Annals of Tourism Research, 31(3), 556-579. Doi: 10.1016/j.annals.2004.01.008

Santos, F.J. y Liñán, F. (2007). Measuring Entrepreneurial Quality in Southern Europe. International Entrepreneurship and Management Journal, 3(1), 87-107. 
Schroeder, T. (2003). Background and Motivations of Resource-Based Tourism Operators in the Northern Great Plains: A Qualitative Study. Retrieved 12th October, 2008. Recuperado de : www.und.edu/instruct/tschroed/nattour1.pdf

Schultz, T. (1985). Invirtiendo en la gente: la cualificación personal como motor económico. Barcelona, España: Ariel.

Sharma, A., y Upneja, A. (2005). Factors influencing financial performance of small hotels in Tanzania. International Journal of Contemporary Hospitality Management, 17(6), 504- 515. doi:10.1108/09596110510612149

Shaw, G. (2003). Entrepreneurial cultures and small business enterprises in tourism. En M. C. Hall, A. Lew, y A. Williams (Eds.), Blackwells companion to tourism geography. Oxford, UK: Blackwells.

Smith, S.L.J. (2006). How big? How many? Enterprise size distributions in tourism and other industries. Journal of Travel Research, 45(1), 53-58. doi:10.1177/0047287506288886

Stanworth, J. y Gray, C. (1991). Entrepreneurship and Education: Action Based Research with Training Policy Implications in Britain. ENDEC. Singapore.

Storey, D. (2000). Six steps to heaven: evaluating the impact of public policies to support small business in developed countries. En D. Sexton y H. Landstrom (Eds.), Handbook of entrepreneurship (pp 176-193). Oxford, UK: Balckwell.

Szivas, E. (2001). Entrance into tourism entrepreneurship: a UK case study. Tourism and Hospitality Research, 3(2), 163-172. doi:10.1177/146735840100300207

Teo, P. y Lim H.L. (2003): Global and local interactions in tourism, Annals of Tourism Research, 30 (2), 287-306. doi:10.1016/S0160-7383(02)00049-X

Thomas, R. (1998). Small firms and the state. En R. Thomas (Ed.), The management of small tourism and hospitality firms. London, UK: Cassell.

Thomas, R. (2000). Small firms in the tourism industry: some conceptual issues. International Journal of Tourism Research, 2(5), 345-353. doi:10.1002/1522-1970(200009/10).

Thomas, R., Shaw, G. y Page, S.J. (2011). Understanding small firms in tourism: A perspective on research trends and challenges. Tourism Management, 32(5), 963-976. doi: 10.1016/j.tourman.2011.02.003

Wanhill, S. (1996). Local enterprise and development in tourism. Tourism Management, 17(1), 35-42. doi:10.1016/0261-5177(95)00098-4

Wanhill, S. (2000). Small and medium tourism enterprises. Annals of Tourism Research, 27(1), 132-147. doi:10.1016/0160-7383(99)00072-9 